\title{
Spatial structure of the plasma sheet boundary layer at distances greater than $180 R_{E}$ as derived from energetic particle measurements on GEOTAIL
}

\author{
D. V. Sarafopoulos ${ }^{1}$, E. T. Sarris ${ }^{1}$, V. Angelopoulos ${ }^{2}$, T. Yamamoto $^{3}$, S. Kokubun ${ }^{4}$ \\ ${ }^{1}$ Demokritos University of Thrace, Department of Electrical and Computer Engineering, Xanthi, GR-67100, Greece \\ ${ }^{2}$ Space Sciences Laboratory, University of California, Berkeley, California, USA \\ ${ }^{3}$ Institute of Space and Astronautical Science, 3-1-1 Yoshinodai, Sagamihara 229, Japan \\ ${ }^{4}$ Solar-Terrestrial Environment Laboratory, Nagoya University, Toyokawa 442, Japan
}

Received: 21 October 1996 / Revised: 9 April 1997 / Accepted: 29 April 1997

\begin{abstract}
We have analyzed the onsets of energetic particle bursts detected by the ICS and STICS sensors of the EPIC instrument on board the GEOTAIL spacecraft in the deep magnetotail (i.e., at distances greater than $180 \mathrm{R}_{\mathrm{E}}$ ). Such bursts are commonly observed at the plasma-sheet boundary layer (PSBL) and are highly collimated along the magnetic field. The bursts display a normal velocity dispersion (i.e., the higher-speed particles are seen first, while the progressively lower speed particles are seen later) when observed upon entry of the spacecraft from the magnetotail lobes into the plasma sheet. Upon exit from the plasma sheet a reverse velocity dispersion is observed (i.e., lower-speed particles disappear first and higher-speed particles disappear last). Three major findings are as follows. First, the tailwardjetting energetic particle populations of the distant-tail plasma sheet display an energy layering: the energetic electrons stream along open PSBL field lines with peak fluxes at the lobes. Energetic protons occupy the next layer, and as the spacecraft moves towards the neutral sheet progressively decreasing energies are encountered systematically. These plasma-sheet layers display spatial symmetry, with the plane of symmetry the neutral sheet. Second, if we consider the same energy level of energetic particles, then the $\mathrm{H}^{+}$layer is confined within that of the energetic electron, the $\mathrm{He}^{++}$layer is confined within that of the proton, and the oxygen layer is confined within the alpha particle layer. Third, whenever the energetic electrons show higher fluxes inside the plasma sheet as compared to those at the boundary layer, their angular distribution is isotropic irrespective of the Earthward or tailward character of fluxes, suggesting a closed field line topology.
\end{abstract}

\section{Introduction}

Although single-point measurements cannot distinguish spatial from temporal processes, in this paper we present evidence for spatial layering of the deep-tail plasma sheet at distances greater than $180 \mathrm{R}_{\mathrm{E}}$.

The plasma-sheet boundary layer (PSBL) is the region between the plasma sheet and the tail lobes, comprised of highly anisotropic ion distributions (Lui et al., 1978; DeCoster and Frank, 1979; Mobius et al., 1980; Spjeldvik and Fritz, 1981; Williams, 1981; Eastman et al., 1984, 1985; Sarafopoulos and Sarris, 1990, 1991a, b). In the distant tail the PSBL represents the layer of reconnected field lines lying between the separatrix and the thermal plasma sheet (Richardson and Cowley, 1985). Tailward-streaming cold ion beams of ionospheric origin at energies less than $1 \mathrm{keV}$ are also frequently observed in the PSBL (Parks et al., 1984; Eastman et al., 1984).

The PSBL fluxes are expected and observed to $\boldsymbol{E} \times \boldsymbol{B}$ drift toward the neutral sheet for a given externally imposed cross-tail electric field $\boldsymbol{E}$ (Sarris and Axford, 1979; Williams, 1981; Andrews et al., 1981; Richardson and Cowley, 1985; Scholer et al., 1987). The cross-tail field causes two streams of different energy ions ejected at zero pitch angles from an acceleration source to drift toward the neutral sheet with different drift $\boldsymbol{V}_{\text {drift }}$, so that the beams become separated in space.

Mobius et al. (1980) performed a case-study of the near-Earth PSBL during substorm recovery. They found that the particle spectra at the central plasma sheet (CPS) were softer than at the PSBL, and that the alpha particle layer was confined to within the proton layer. Their result will be extended in this work for the distanttail plasma sheet.

Close to Earth, although highly anisotropic angular distributions are often encountered at the PSBL, such energetic particle populations are typically observed at very low intensities relative to the CPS (Sarafopoulos 
and Sarris, 1990, 1991a, b). Only in one exceptional case (Sarafopoulos and Sarris, 1991a) at $\sim 37 \mathrm{R}_{\mathrm{E}}$ downtail, did the higher fluxes of energetic protons occur at the PSBL. Conversely, in the distant plasma sheet (as we shall see in this work), it is not uncommon that peak fluxes of streaming particles occur at the PSBL. In such cases, the fluxes are associated with open magnetic field topology.

Richardson and Cowley (1985) examined tailwardstreaming ion bursts extending into the lobe for a few minutes before entry into or after exit from the plasma sheet in the deep geomagnetic tail. They showed evidence at the leading edge of a normal velocity dispersion (i.e., high-energy particles are detected before low-energy ones), whereas at the trailing edge a reverse dispersion was detected. Such features have been recognized as the result of spatially separated fluxes. Similarly, Scholer et al. $(1986,1987)$ and Scholer (1986) found that the velocity dispersion effect during the appearance and disappearance of the beams in the deep PSBL indicates that these beams are not due to temporal effects. Our work concurs with these authors' conclusions and goes further to demonstrate the substructure of the distant-tail PSBL using GEOTAIL data sets.

\section{Observations and data analysis}

We selected three intervals for thorough study. The first interval encompasses seven successive PSBL crossings and helps demonstrate the symmetry of the energetic particle layers with respect to the neutral sheet. The intervals are 16:00-19:30 UT on 14 March (day 73) 1993, and 08:28-08:43 and 14:25-14:45 UT on 18 April (day 108) 1994. We used data from the magnetic-field (MGF) experiment (Kokubun et al., 1994) at 3-s resolution and the energetic particle (EPIC) instrument (Williams et al., 1994). EPIC consists of sensors ICS and STICS, and the time resolution is declared in each plot varying from 3 to $96 \mathrm{~s}$. All the EPIC measurements are depicted as differential fluxes.

\subsection{Overview of studied interval: 14 March (day73) 1993}

An overview plot of the period 16:00-19:30 UT on 14 March 1993 is presented in Fig. 1. At 18:00 UT the GEOTAIL spacecraft was located at $(X, Y, Z)=(-177$, 35.4, 4.6) $\mathrm{R}_{\mathrm{E}}$ in GSE coordinates. The third panel shows the $B_{x}$ and $B_{z}$ components of the vector magnetic field in GSM coordinates. It is obvious that during this interval the entire magnetotail apparently moved northward and then southward, causing GEOTAIL to pass from the northern lobe into the plasma sheet (16:52 UT), then into the southern-hemisphere lobe (17:24), and finally back into the northern lobe via the plasma sheet (18:10-18:25 UT). A brief excursion of the spacecraft into the CPS $(17: 49-17: 55$ UT) and a final entry into the plasma sheet

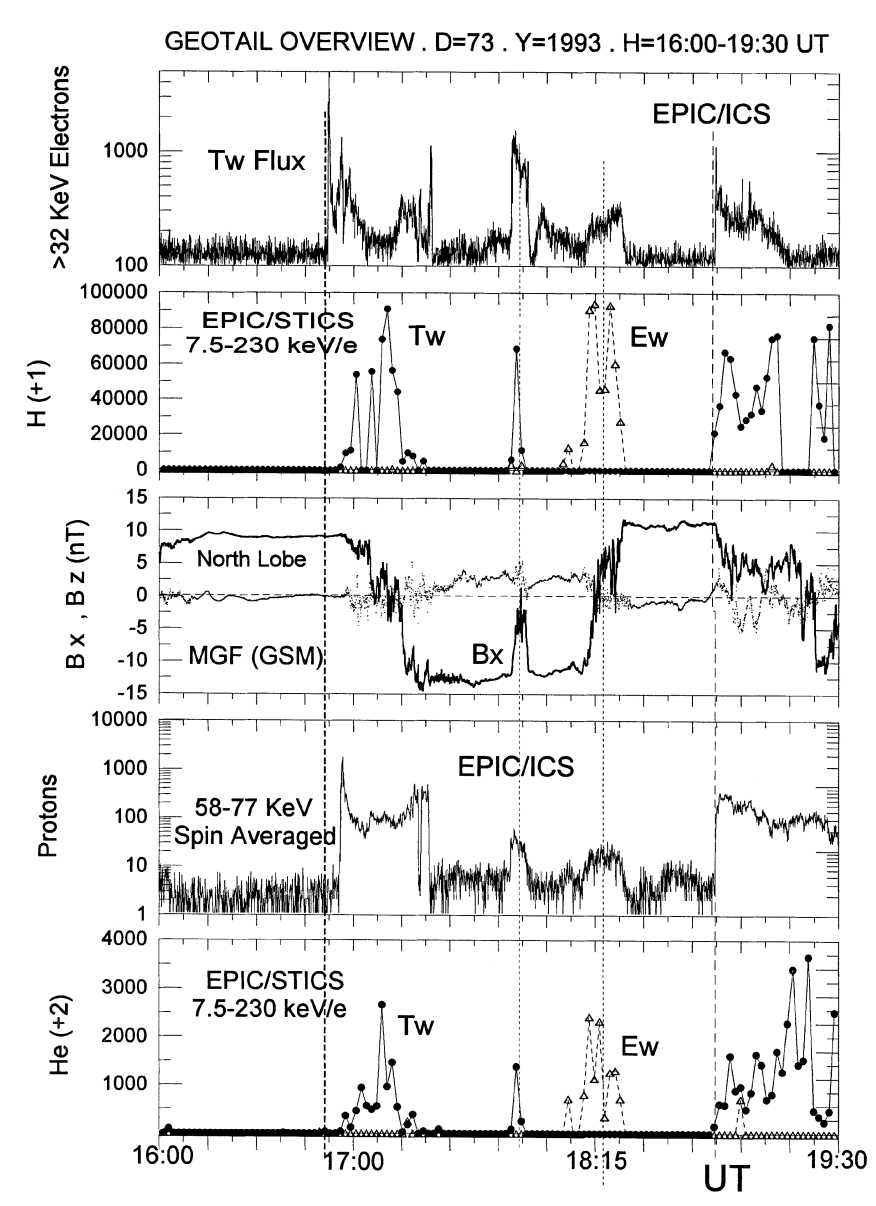

Fig. 1. Overview of the interval 16:00-19:30 UT on day 73, 1993, at $X=-177 \mathrm{R}_{\mathrm{E}}$. Central panel: the $B_{x}$ (solid thick line) and $B_{z}$ (dashed line) components of the magnetic field in GSM coordinates at 3-s resolution. First panel: tailward (Tw) flux (which is measured by the sunward-looking sector) of $>32-\mathrm{keV}$ electrons. Second and bottom panels: Tw (solid dotted line) and earthward (Ew) fluxes of the 7.5$230-\mathrm{keV} / \mathrm{e}$ protons and $\mathrm{He}^{++}$, respectively, of the EPIC/STICS sensor. Fourth panel: spin-averaged $58-77-\mathrm{keV}$ energetic protons of the EPIC/ICS sensor. All the particle data are depicted as differential fluxes

(18:52 UT) are also notable. The $\mathrm{H}^{+}$and $\mathrm{He}^{++}$ differential intensities of the EPIC/STICS data sets (second and fifth panels) reveal prevailing tailward fluxes (solid circles), as well as the existence of an interval of intense Earthward flux (open triangles) simultaneously with the second passage throughout the plasma sheet. Energetic $(>32 \mathrm{keV})$ electrons of the EPIC/ICS data are shown in the first panel, demonstrating that higherstreaming fluxes occurred preferentially at the plasma sheet adjacent to the lobe magnetic field or on lobe field lines. The same is valid with all species of energetic fluxes, and this will become clearer in the next paragraphs through a systematic study of all the spacecraft transitions. A representative flux of 58-77$\mathrm{keV}$ energetic protons is displayed in the fourth panel, and a discernible time-lag of $\sim 4 \mathrm{~min}$ is apparent between electrons and protons for the first detected onsets of fluxes. 


\subsection{First passage through the plasma sheet (16:30-17:45 UT)}

During this interval, shown in Fig. 2, the spacecraft traversed the plasma sheet from the northern to the southern lobe. The neutral-sheet traversal is evidenced by the sign change in the $x$-component of the magnetic field (third panel, thick line). The energetic electrons (first panel) stream on lobe field lines or at the PSBL region. The ratio of field-aligned tailward fluxes to Earthward fluxes of electrons of the EPIC/ICS data is given in the third panel (thin line, $\log$ scale). It is apparent that the beam-like electron distributions (which are the unique feature of an open magnetic field line topology) are followed by nearly isotropic electron distributions (i.e., closed magnetic field lines). The energetic 194-282-keV alpha particles (second panel), as well as the energetic $58-77-\mathrm{keV}$ protons (fourth and fifth panels), show a time-delay of $4 \mathrm{~min}$ in their onset relative to electrons. They mainly stream tailward at the PSBL adjacent to the lobe. Conversely, the lowestenergy alpha particles measured $(9.4 \mathrm{keV})$ (second panel) as well as protons (bottom panel) essentially convect

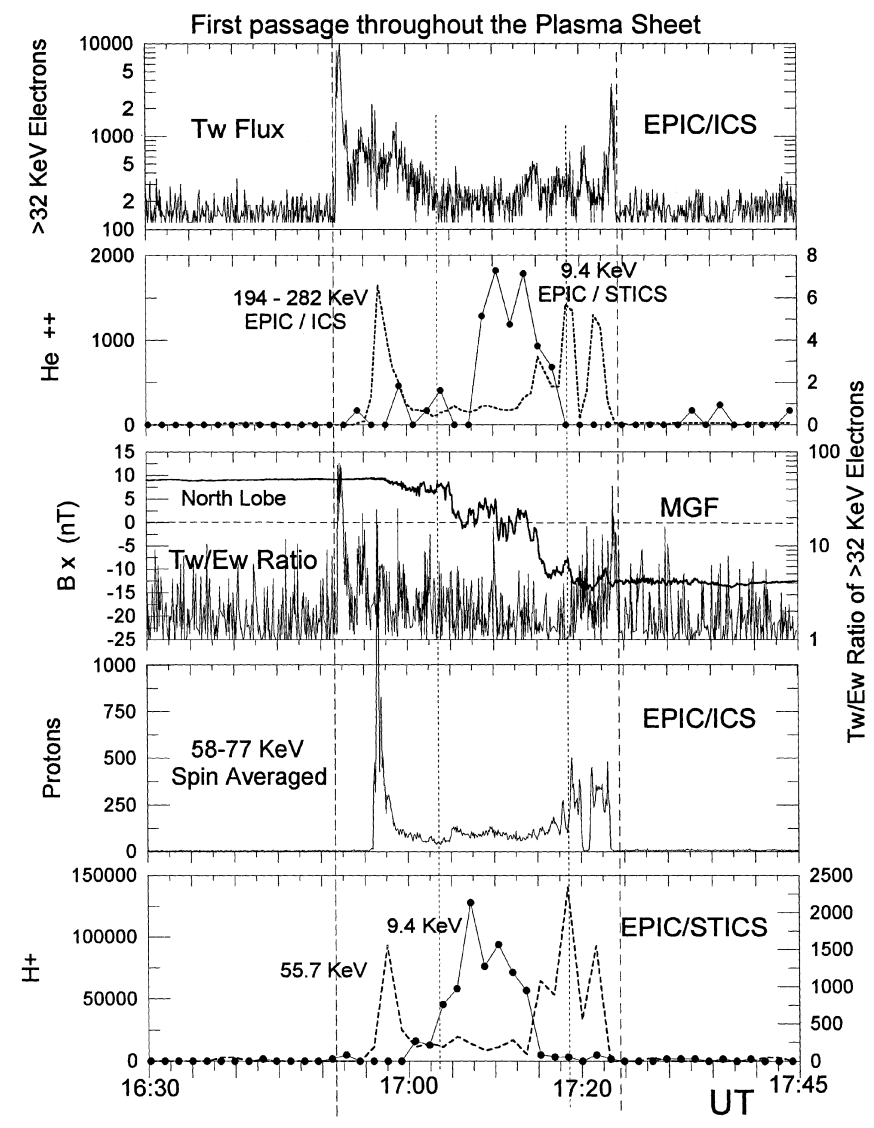

Fig. 2. An expanded view of the first transition from the north to the south lobes (16:30-17:45 UT) shown in Fig. 1. The central panel shows the $B_{x}$ component of the magnetic field (thick line) as well as the Tw/Ew ratio of $>32-\mathrm{keV}$ differential fluxes of electrons (thin line). The second and bottom panels give the $\mathrm{He}^{++}$and proton fluxes, respectively, at the same time for the lowest measured energy level ( $\sim 9.4 \mathrm{keV}$, solid lines) and the much higher one (greater than $55 \mathrm{keV}$, dashed lines)

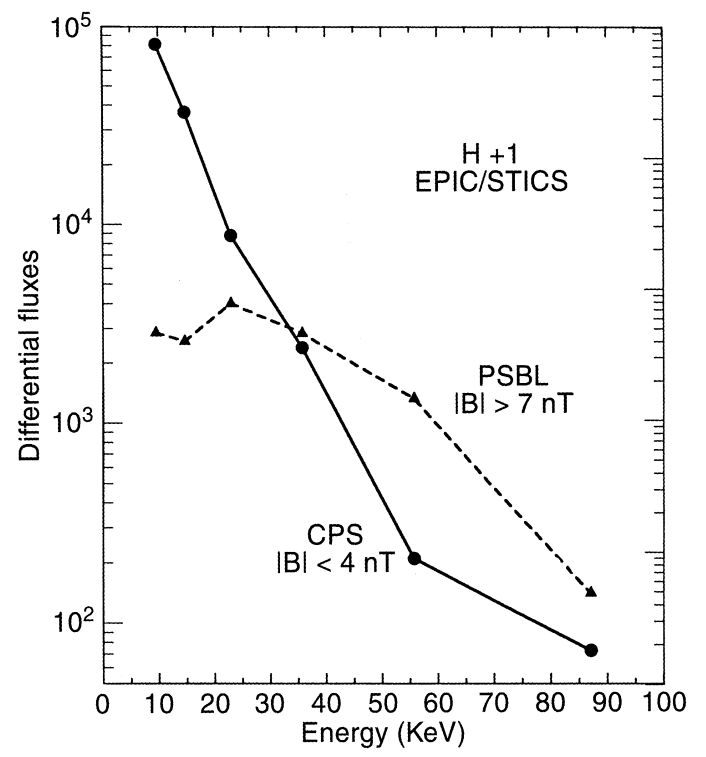

Fig. 3. The central plasma sheet shows a softer spectrum for energetic protons as compared to that at the plasma sheet boundary layer. This figure is derived from data corresponding to the interval 17:05-17:25 UT of Fig. 2

only at the CPS region (where the $B_{x}$ is significantly depressed and the energetic electrons are isotropic).

The distinct spatial separation among high- and lowenergy protons (shown at the bottom panel of Fig. 2) is further illustrated with the help of energy spectra shown in Fig. 3. The PSBL and CPS are identified as the layers where the magnetic field magnitude is greater than $7 \mathrm{nT}$ and less than $4 \mathrm{nT}$, respectively. The proton energy spectrum at the CPS on the basis of EPIC/STICS measurements is softer than that at the PSBL. An explanation for the softening of the spectrum is given in terms of a cross-tail electric field causing different energy beams of protons to drift inward with the same speed, so that the beams become separated in space.

The spatial structure of the plasma sheet is studied in detail in Fig. 4a, which corresponds to the GEOTAIL transition from the north lobe to the CPS. We observe in good order time-delayed peak fluxes of energetic protons (tailward fluxes with 24- or 48-s resolution) relative to energetic electrons (tailward fluxes with 3-s resolution). The energies of protons continuously decrease toward the bottom panel from superthermal to thermal population (only $9.4 \mathrm{keV}$ ). It is evident that the plasma sheet is itself composed of successive layers of ejected particles of decreasing energy as the spacecraft moves from the separatrix and approaches the CPS. Figure $4 \mathrm{~b}$ shows in detail the reverse velocity dispersion detected as the spacecraft moved from almost the neutral sheet to the south lobe. The peak flux at 9.4$\mathrm{keV}$ protons occurred at the innermost depths of the plasma sheet (see also Fig. 2). This reverse dispersion can be interpreted only in terms of spatial separation of the ejected particles from the acceleration source. In addition, the preceding observations imply that the plasma sheet displays a symmetric structure with the neutral sheet being the plane of symmetry. 

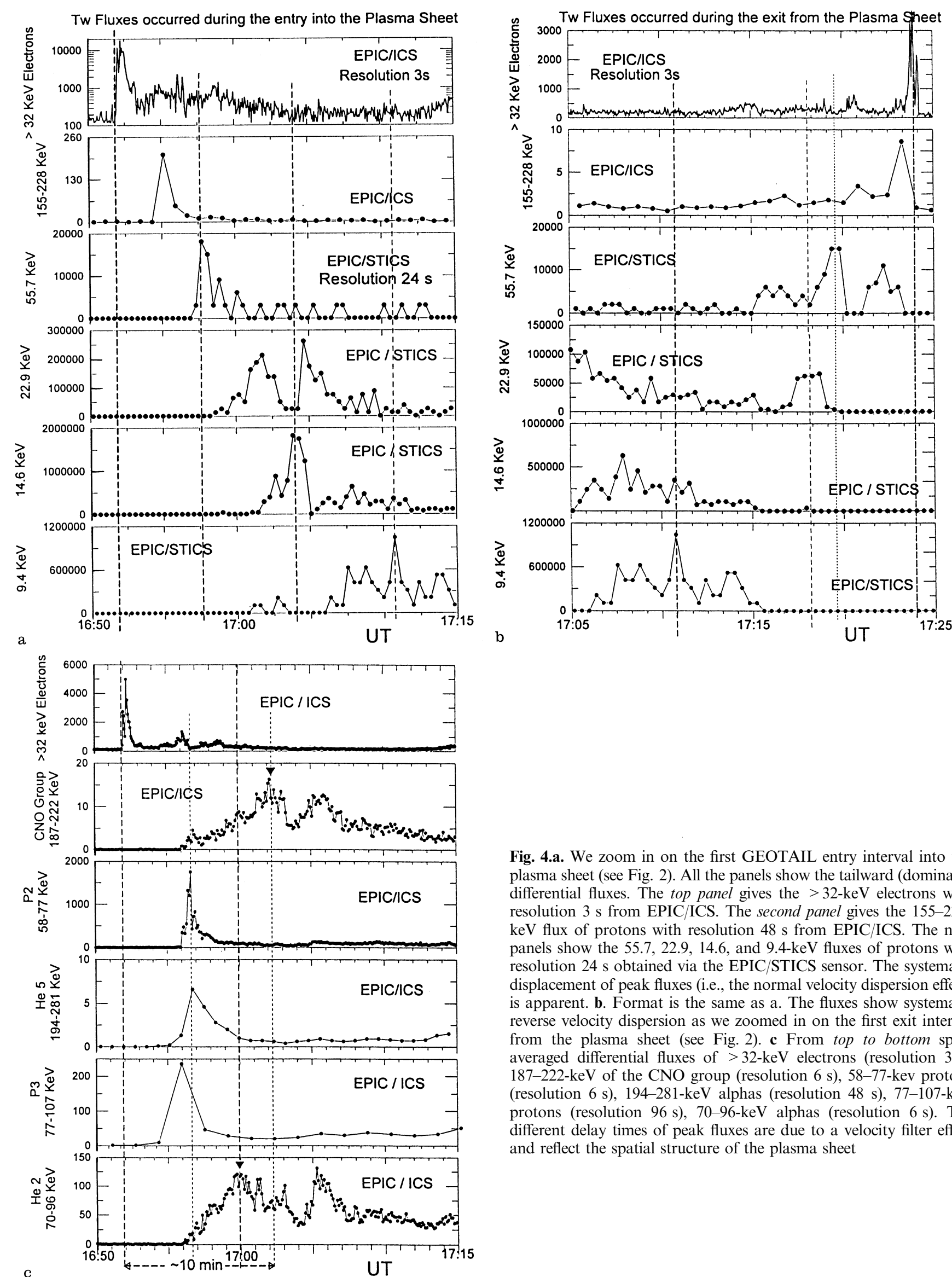

Fig. 4.a. We zoom in on the first GEOTAIL entry interval into the plasma sheet (see Fig. 2). All the panels show the tailward (dominant) differential fluxes. The top panel gives the $>32-\mathrm{keV}$ electrons with resolution $3 \mathrm{~s}$ from EPIC/ICS. The second panel gives the 155-228$\mathrm{keV}$ flux of protons with resolution $48 \mathrm{~s}$ from EPIC/ICS. The next panels show the 55.7, 22.9, 14.6, and 9.4-keV fluxes of protons with resolution $24 \mathrm{~s}$ obtained via the EPIC/STICS sensor. The systematic displacement of peak fluxes (i.e., the normal velocity dispersion effect) is apparent. b. Format is the same as a. The fluxes show systematic reverse velocity dispersion as we zoomed in on the first exit interval from the plasma sheet (see Fig. 2). c From top to bottom spinaveraged differential fluxes of $>32-\mathrm{keV}$ electrons (resolution $3 \mathrm{~s}$ ), 187-222-keV of the CNO group (resolution $6 \mathrm{~s}$ ), 58-77-kev protons (resolution $6 \mathrm{~s}$ ), 194-281-keV alphas (resolution $48 \mathrm{~s}$ ), 77-107-keV protons (resolution $96 \mathrm{~s}$ ), 70-96-keV alphas (resolution $6 \mathrm{~s}$ ). The different delay times of peak fluxes are due to a velocity filter effect and reflect the spatial structure of the plasma sheet 
Figure $4 c$ further exemplifies the effect of velocity dispersion using different species. It is evident that:

1. When the alpha particles have almost four times the energy of protons then the same arrival time is anticipated for both. This is the situation displayed in third and fourth panels.

2. The bottom two panels show that the alpha particles lag in arrival relative to protons, and this is anticipated because both of them have nearly equal total energies. Therefore, the alpha layer is confined to within that of the protons.

3 . The delay time of the $\sim 187-\mathrm{keV}$ oxygen particles relative to electrons is $10 \mathrm{~min}$ and that of the $\sim 70$ $\mathrm{keV}$ alphas is $8 \mathrm{~min}$ (i.e., ratio 1.25) in expected relative delay based on the speeds of these species (i.e., ratio 1.22 ).

4. The $\sim 58-\mathrm{keV}$ protons and the $\sim 70-\mathrm{keV}$ alpha particles (third and sixth panels in Fig. 4c) give 4- and 8min observational delay times, respectively, relative to electrons. The ratio of these delays is very close to the theoretically expected ratios for the species (that is, 0.548).

Thus, on the basis of a, b and c Fig. 4 the plasma sheet could be regarded as "highly stratified with a multitude of successive layers". Certainly the word "layer" here corresponds to the number of our channels. As a matter of fact, the particles are spatially ordered according to a velocity filter effect. The velocity filter effect results in a continuous splitting of particles originating from a source producing particles with different velocities. Nevertheless, a sketch of five successive energy-dependent layers are schematically shown in Fig. 5 in association with the strength of the local magnetic field. The distance scale in figure is arbitrary, because we fail to determine the velocity of the spacecraft relative to the PSBL (that would be derived from gradient anisotropy of energetic particles). Indicative angular distributions of the energetic particles at the PSBL as well as at the CPS are displayed and discussed later on.

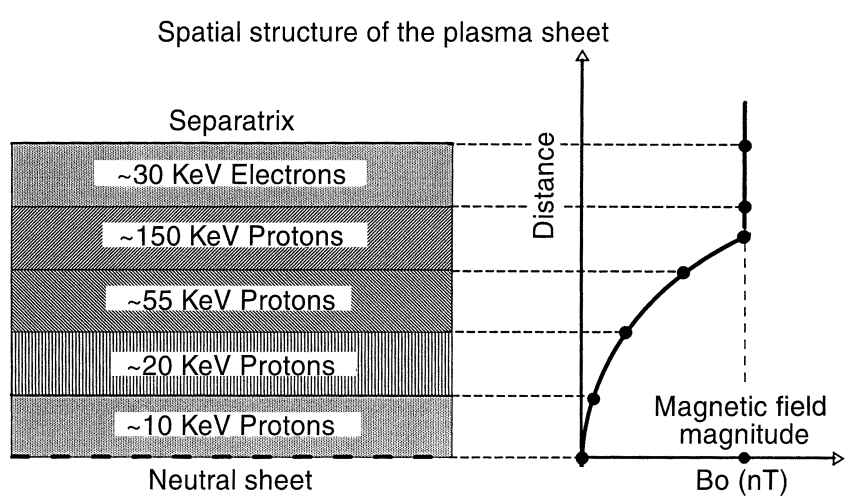

Fig. 5. Sketch illustrating the spatial structure of the plasma sheet. Five successive layers (dependent on energy and species) are encountered as the spacecraft crosses the separatrix moving toward the neutral sheet. Here, the energetic electrons stream exclusively on lobe field lines (characterized by constant magnitude of the lobe magnetic field). The distance scale is arbitrary

\subsection{A brief excursion from south lobe toward the neutral sheet (17:49-14:55 UT)}

Figure 6 shows a gradual GEOTAIL excursion from the south-lobe magnetic field toward the CPS (see third panel). In general, we observe that the higher fluxes of energetic electrons (first panel), as previously, occurred at higher magnetic field values, whereas the more highly energetic ion intensities detected essentially with relatively lower magnetic field magnitudes and therefore closer to the neutral sheet. The electrons display tailward beam-like angular distributions adjacent to the lobe plasma sheet, while at the CPS regions they are isotropic. The differentiation between tailward (Tw) and Earthward (Ew) fluxes is denoted with solid-dotted and dashed lines, respectively. The $35-46.8-\mathrm{keV}$ ions show a $\sim 30$-s lag in onset, while they lead the way by $60 \mathrm{~s}$ in exiting the plasma sheet. This difference could be associated with the more gradual entry into the plasma

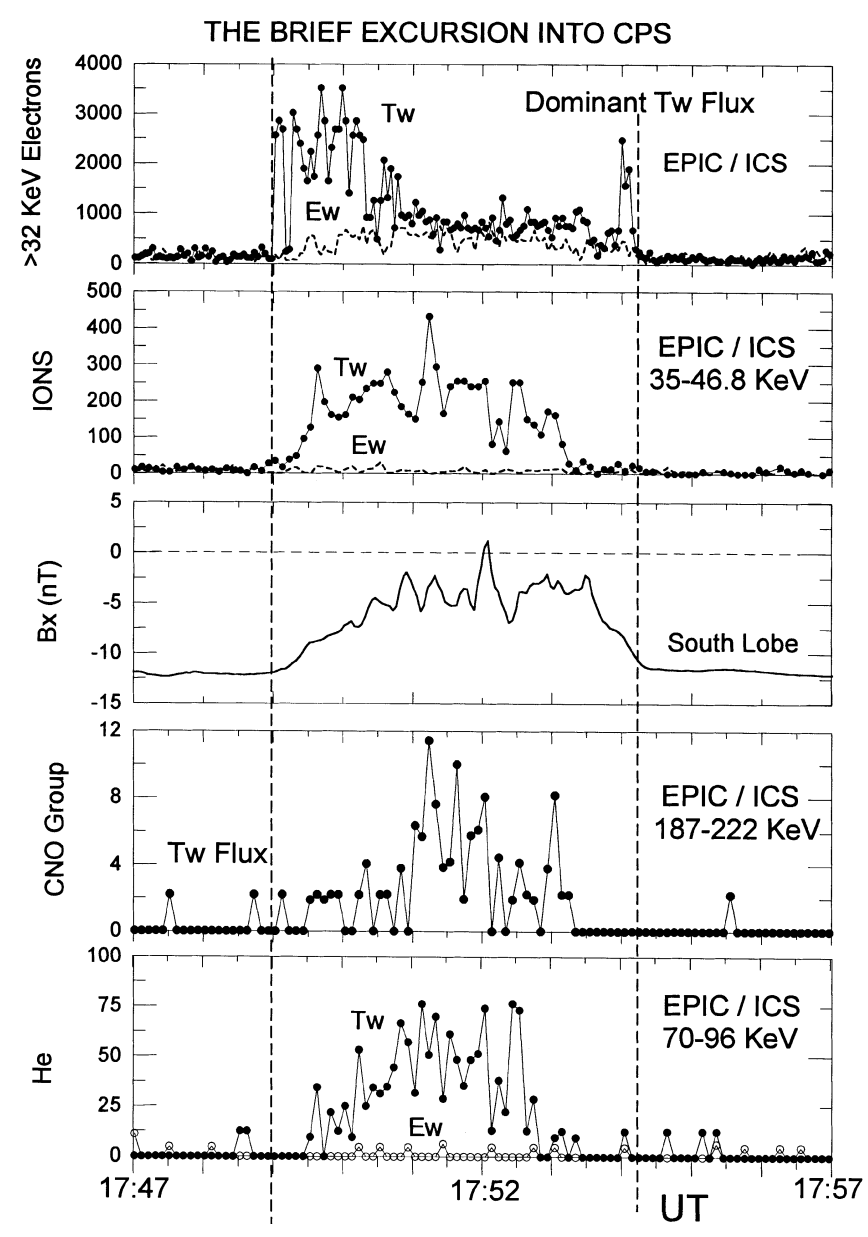

Fig. 6. A GEOTAIL brief excursion from the south lobe toward the CPS and then back to the lobe structure, as it is seen along the magnetic field $B_{x}$ component at the central panel. The $>32-\mathrm{keV}$ electrons (first panel) stream tailward (solid dotted line) at the PSBL regions, whereas they show isotropic character at the CPS one. The tailward flux dominates along the $35-46.8-\mathrm{keV}$ ion trace (second panel), the 187-222-keV CNO group trace (fourth panel) and the 7096-keV trace of He particles (bottom panel). The abrupt exit from the plasma sheet is accompanied by short-lived energetic electron fluxes 
sheet (compared to the exit from it). The oxygen particles (fourth panel) occur only on the innermost layer of the plasma sheet.

\subsection{A second transition from the south} to the north lobe: (18:10-18:25 UT), Earthward fluxes at $\sim 180 R_{E}$

During the interval 18:10-18:25 UT, GEOTAIL moved from the south to the north lobe. The prominent feature throughout this plasma-sheet crossing is the prevailing Earthward flux of all the ion species. The third panel of Fig. 7 illustrates this transition evidenced in the $B_{x}$ component of the magnetic field. No significant net Earthward or tailward flux of energetic electrons is detected (first panel), which is indicative of a closed topology of magnetic field lines. An additional striking feature associated with the energetic electrons is that the more intense fluxes occurred almost at the CPS, a feature similar to the ion fluxes (second panel). In this case, the trapped electrons have the required time to drift toward the CPS as they remain within the closed field line geometry of magnetic field lines. The duration of Earthward flux is $12 \mathrm{~min}$. If we assume the nearEarth neutral-line model for substorms, then according to this model the flow reversal detected at $\sim 180 \mathrm{R}_{\mathrm{E}}$ may correspond to the tailward retreat of the near-Earth reconnection region downstream of the spacecraft.

\subsection{The last entry into the plasma sheet (18:52 UT)}

At $\sim 18: 52$ UT the GEOTAIL spacecraft entered the plasma sheet, while the dominant flux of energetic ions and electrons was tailward. The trace of the third panel of Fig. 8 displays the $B_{x}$ component of the magnetic field and displays a gradual transition toward the neutral sheet. Higher differential intensities of energetic electrons with more anisotropic distributions occurred at relatively larger values of the magnetic field. Three local maxima marked as (a), (b), and (c) along the $B_{x}$

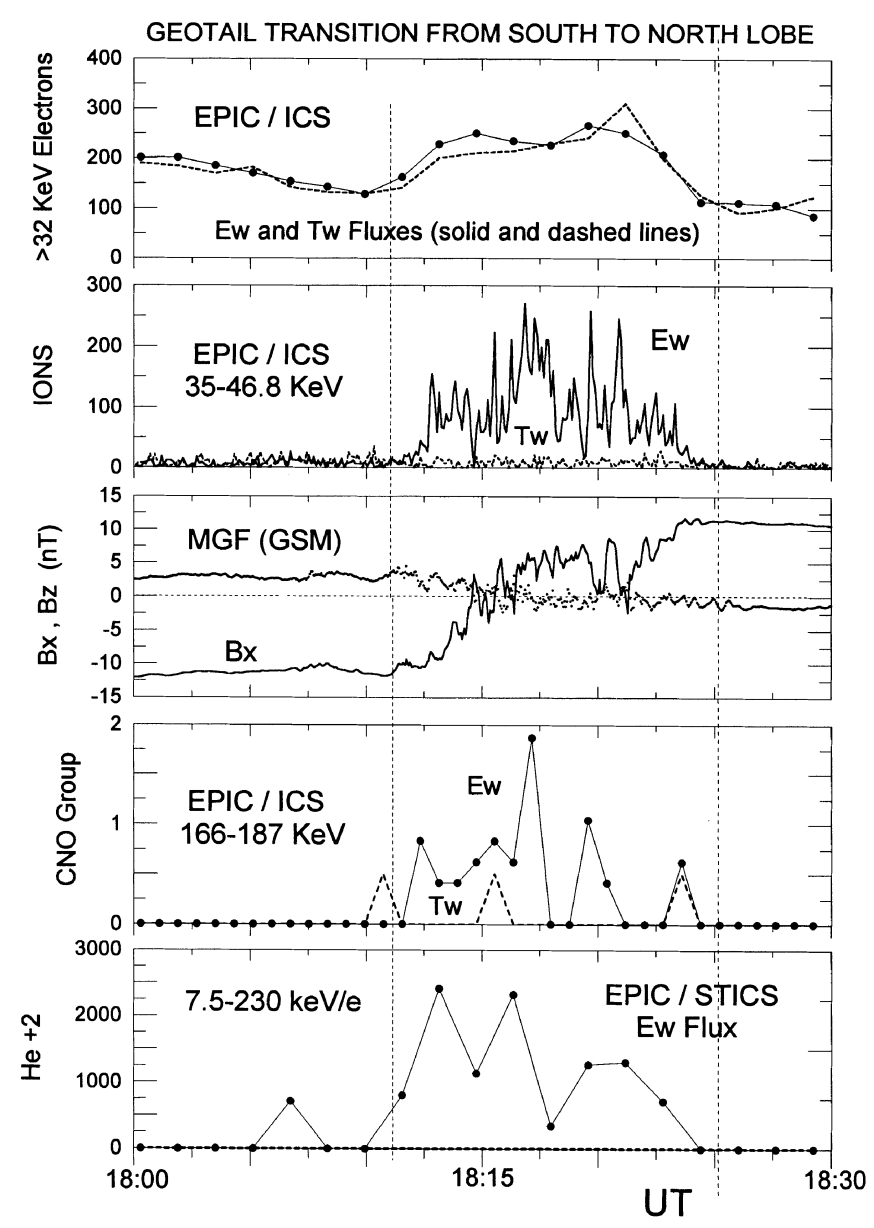

Fig. 7. The GEOTAIL transition from the south to the north lobe magnetic field structure (central panel, $B_{x}$ component) is associated with almost isotropic $>32-\mathrm{keV}$ electron fluxes (first panel). The dominant Earthward fluxes of the 35-46.8-keV ions (second panel), the 166-187-keV CNO group ions (fourth panel) and 7.5-230-keV/e alphas (bottom panel) are apparent. The electrons do not display peak fluxes at the PSBLs

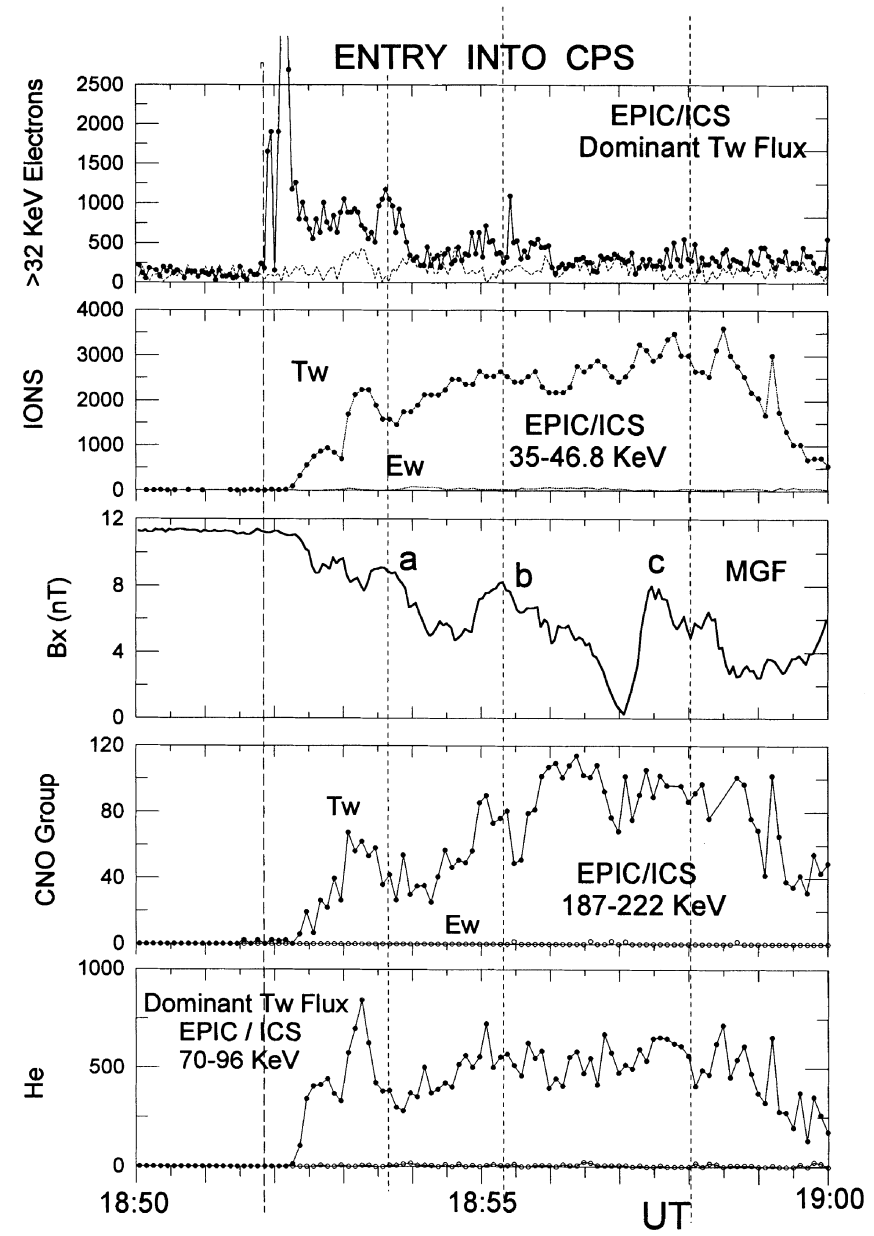

Fig. 8. Final entry into the plasma sheet with obvious normal velocity dispersion effect. The energetic electrons stream tailward at the PSBL region (first panel), while all the other ions show peak fluxes at inner layers of the plasma sheet. The three local maxima marked as (a), (b), and (c) along the magnetic field trace (third panel) correspond to higher and more anisotropic energetic electron fluxes 
trace corroborate this statement. On the other hand, higher fluxes of energetic ions are mainly confined within the CPS. The arrival time of the energetic ions was delayed by $\sim 30 \mathrm{~s}$ compared to the onset of the energetic electrons.

\subsection{Additional examples of bursts with dispersive onsets}

Two more examples of dispersive onsets of energetic particle bursts, which occurred on 18 April (day 108) 1994 are shown in Figs. 9 and 10. The two figures correspond to an entry into and an exit from the plasma sheet, respectively. In the former case a normal velocity dispersion in peak fluxes is apparent and spans a period of $\sim 10 \mathrm{~min}$, whereas in the latter case a reverse velocity dispersion covers a period of more than $4 \mathrm{~min}$. On both cases all the species stream tailward. The spacecraft was located at $(X, Y, Z)_{\mathrm{GSM}}=(-197.6,21.5,1.8)$ and $(X$, $Y, Z)_{\mathrm{GSM}}=(-197.5,22.1,1.5)$ during the intervals of Figs. 9 and 10, respectively.

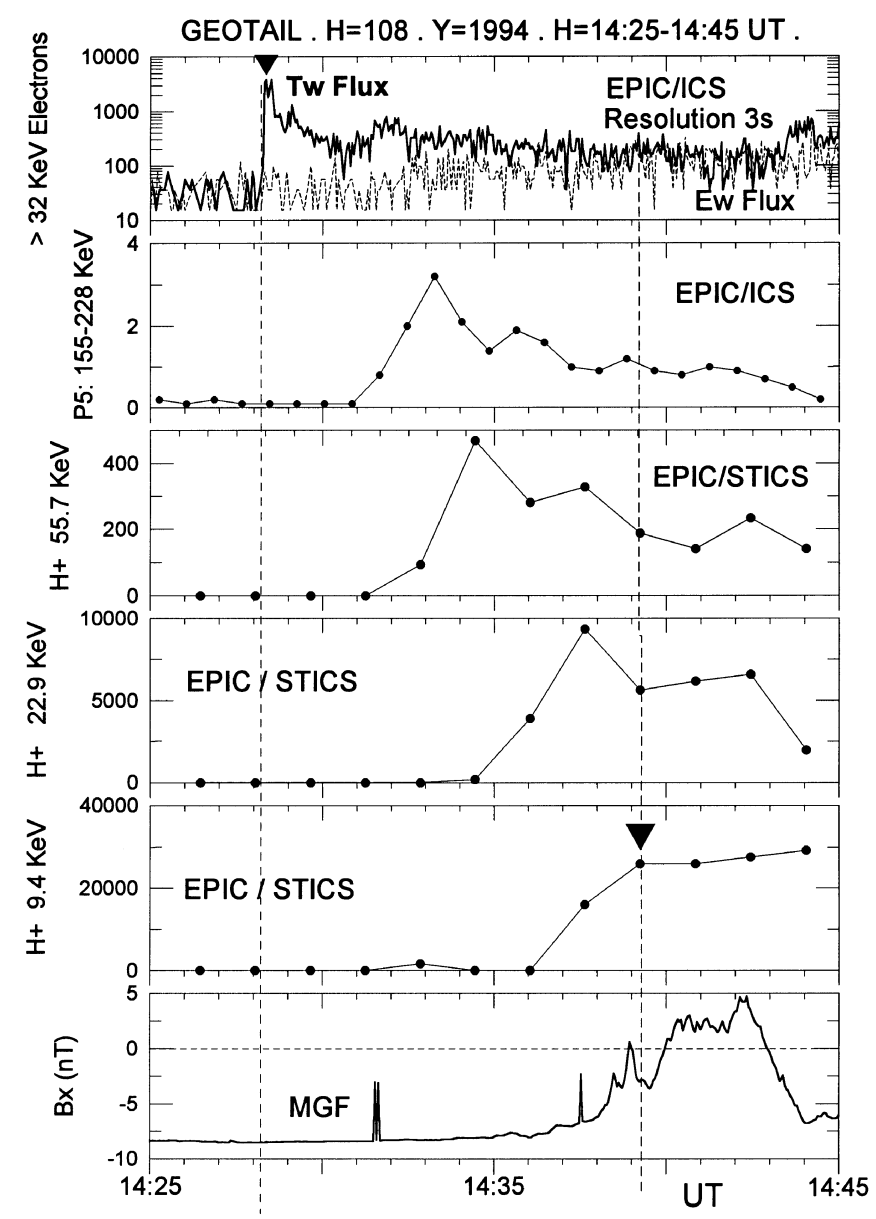

Fig. 9. As the GEOTAIL spacecraft enters the plasma sheet (see the $B_{x}$ magnetic field component at the bottom panel), it detects a systematic displacement of peak fluxes. First appear the tailwardstreaming $>32-\mathrm{keV}$ electrons, then arrive the fastest protons (155$228 \mathrm{keV}$, second panel) and progressively lower energy protons layers are encountered with mean energies 55.7, 22.9, and $9.4 \mathrm{keV}$, corresponding to the third, fourth, and fifth panels

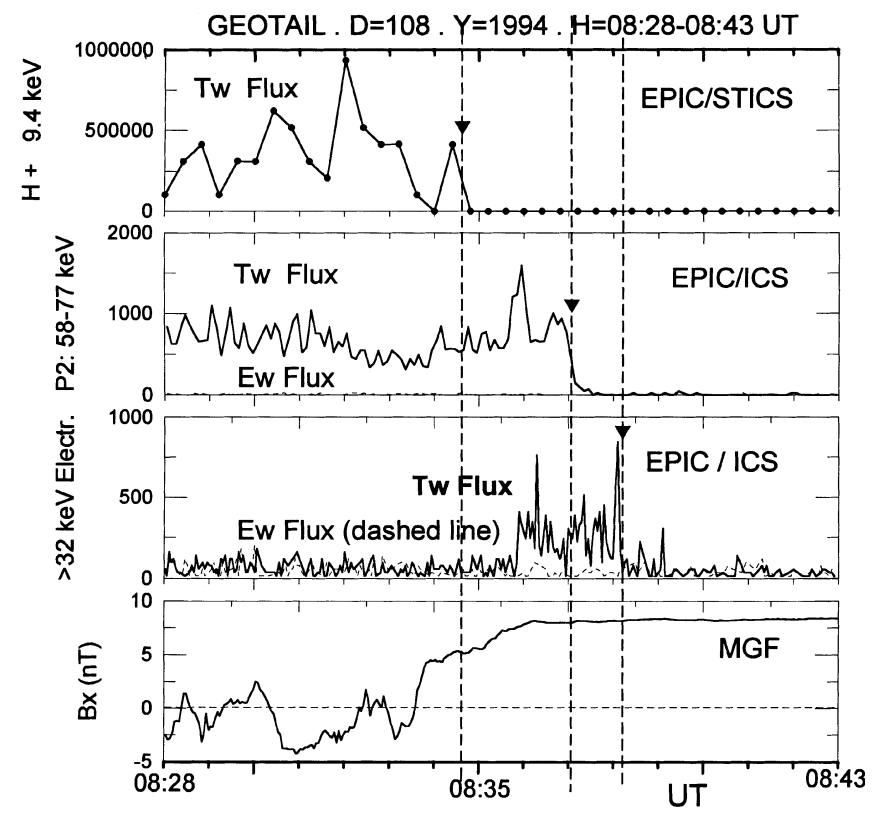

Fig. 10. GEOTAIL exits the plasma sheet to the north lobe magnetic field structure (bottom panel) and detects a clear reverse velocity dispersion effect. First the lower-energy protons disappear $(\sim 9.4 \mathrm{keV}$, first panel) and finally the $>32-\mathrm{keV}$ energetic electrons (third panel). The dominant flux is the tailward (solid thick line)

\section{Discussion}

The main purpose in this article is to establish the highly stratified spatial structure of the distant plasma sheet with few representative examples. Our results confirm previous ISEE-3-examined velocity dispersions also interpreted as spatial features. The temporal resolution, species differentiation, and multiplicity of channels of the EPIC instrument provide us with a further insight into the problem of structure and dynamics of the Earth's magnetotail. Indeed, in the foremost case-study of this work, the spatial structure of the plasma sheet is monitored in successive snapshots, as an active X-line was possible propagating over the spacecraft. The successive entries into and exits from the plasma sheet (seven crossings) associated with normal or reverse velocity dispersions, give strong evidence of a quasipermanent plasma-sheet structure for at least $2.5 \mathrm{~h}$.

Had the detected velocity dispersions been considered a result of varying times of flight of the different velocity particles ejected from an X-line, the following problems would have arised:

1. The reverse velocity dispersion during exits from the plasma sheet would not be explainable by the same process.

2. A smooth and gradual entry into the plasma sheet is associated with sustained fluxes of energetic electrons (see $~ 17: 10$ UT in Fig. 6). Instead, an abrupt exit is associated with short-lived fluxes. In the latter case the electron layer is traversed briefly (see $\sim 17: 54$ UT in Fig. 6). 
3. Local peaks of energetic electrons are observed to be well associated with local magnetic field increases showing the spacecraft approach to the PSBL.

4. Successive peaks of fluxes of tailward-streaming electrons occur systematically and exclusively adjacent to the lobe plasma sheet or on lobe field lines, while the spacecraft traverses the whole plasma sheet.

5. The low-energy particles (i.e., $9.4 \mathrm{keV}$ ) occur in essence at almost zero magnetic field magnitudes.

Richardson and Cowley (1985) examined strongly tailward-streaming ion bursts extending into the lobe for a few minutes after exit from the plasma sheet at the deep geomagnetic tail. They found evidence at the leading edge a normal velocity dispersion (i.e., highenergy particles are detected before low-energy ones), whereas at the trailing edge a reverse dispersion was detected, and such features have been recognized as the result of spatially separated fluxes. Similarly, Scholer et al. (1987) found that the velocity dispersion effect during the appearance and disappearance of the beams in the deep PSBL indicates that these beams are not due to temporal effects. Indeed, this work agrees with such a conclusion and goes further to demonstrate with an improved data set the substructure of the PSBL in the distant plasma sheet. Parks et al. (1992) examined the PSBL crossings close to the Earth $\left(\sim 20 \mathrm{R}_{\mathrm{E}}\right.$ downtail $)$ at 0.16 -s resolution, and demonstrated that the proton and electron boundaries of energies $2-6 \mathrm{keV}$ are displaced in time. This feature was interpreted as indicative of a genuine spatial structure not caused by the different Larmor radii of electrons and protons, since then the ions would be extended further into the lobe. Instead, what was observed was that the electron boundary is extended further out into the lobe.

At the PSBL close to the Earth, it is also well documented that although highly anisotropic angular distributions are often encountered, nevertheless in the vast majority of the cases they are associated with extremely low energetic particle intensities (Sarafopoulos and Sarris, 1990, 1991a,b). Only one exceptional case-study has been presented where at $\sim 37 \mathrm{R}_{\mathrm{E}}$ downtail simultaneous beam-like angular distributions at the PSBL and higher fluxes of energetic ions compared to those at the CPS were observed (Sarafopoulos and Sarris, 1991a). The situation is different for the PSBL at distances greater than $180 \mathrm{R}_{\mathrm{E}}$, as we have concluded in the present article. The deep-tail PSBL frequently displays peak fluxes of energetic ions and electrons, while weaker fluxes occur as the spacecraft moves inward to innermost depths of the plasma sheet. In this work the majority of PSBL crossings associated with tailward-streaming population comply with such a result. However, PSBL crossings exhibiting the opposite behavior are also detected. The distinct feature of the latter crossings is that the higher fluxes are associated with isotropic electrons. And in such a closed topology of the magnetic field the trapped particles have all the required time to convect inward, creating peak fluxes at the CPS (see Fig. 7). To support observationally the last statement we present an additional striking case asso-

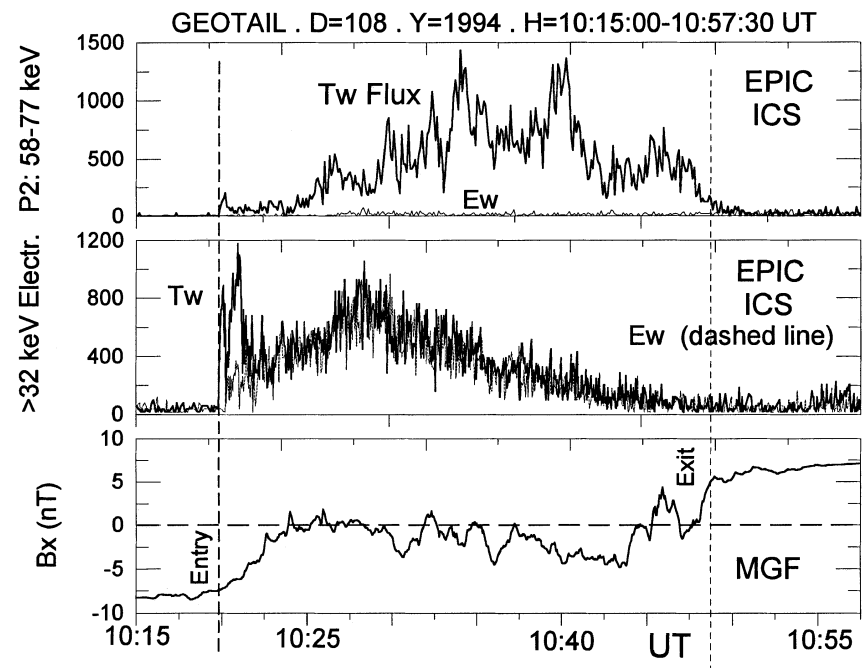

Fig. 11. The $>32-\mathrm{keV}$ energetic electrons show two peak fluxes (second panel). The first occurred during the entry into the plasma sheet (third panel) and displays tailward flux with beam-like angular distributions, while the second occurred at the CPS and shows isotropic electron fluxes. The $58-77-\mathrm{keV}$ protons (first panel) convect tailward (thick line)

ciated with Fig. 11. In this example the more intense fluxes of energetic 58-77-keV protons occurred at the CPS but with isotropic electrons. A close examination of Fig. 11 reveals that although the energetic protons display a tailward flux, the energetic electrons are isotropic. Conversely, at 10:20 UT the high fluxes of energetic electrons that occurred at the PSBL are associated with highly anisotropic angular distributions.

In the near-Earth magnetotail the direction of the bulk plasma flow, as well as the energetic particle fluxes, varies considerably. Conversely, in the deep magnetotail (distances greater than $150 \mathrm{R}_{\mathrm{E}}$ ) the tailward population becomes the dominant characteristic, while Earthward flows occurred in less than $1 \%$ of all cases (Zwickl et al., 1984; Daly et al., 1984; Schindler et al., 1989). In the present work the reversal to Earthward fluxes of all ions observed at $\sim 180 \quad \mathrm{R}_{\mathrm{E}}$ downtail (for an interval of $\sim 12$ min on 14 March 1993) could be well interpreted in terms of a neutral line retreating tailward over the spacecraft. Figure 12 presents common-scale (200 nT per division) ground magnetograms from auroral stations. On $x$-component traces substorms can be identified by sharp negative perturbations (westward electrojets) or by positive excursions (eastward electrojets). Critical in the identification of substorm onset is the local time of the station. Only stations that are close to midnight $( \pm 2 \mathrm{~h}$ of local time) can be used for substorm identification. Digital ground magnetometer coverage is not very good in the Russian sector and therefore an accurate determination of substorm onset based on the limited number of nightside stations between 15:00 and 23:00 UT is not always possible. At $\sim 16: 48$ UT a negative bay onset at the Russian station TIX started, and this time could be considered as the substorm onset given that for the Tixie station the MLT $=0$ occurs at 15:37 UT. At the same time the 


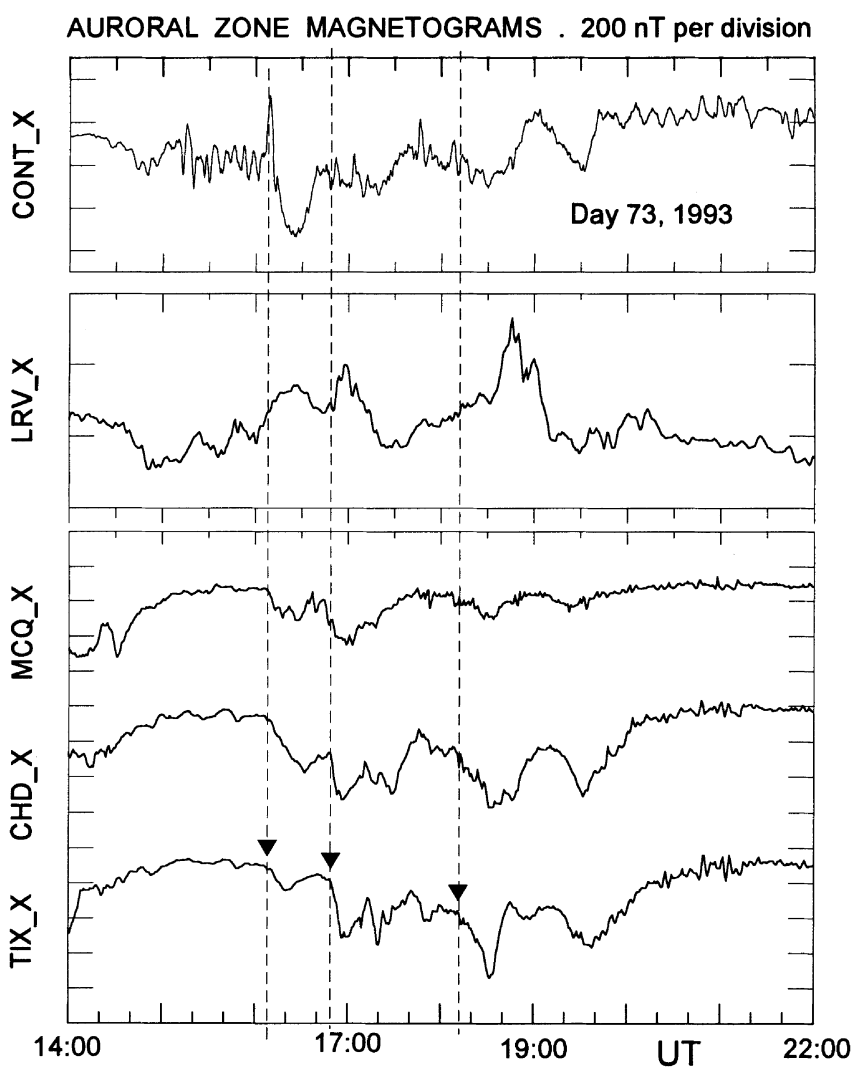

Fig. 12. Recordings of common-scale magnetometer data (200 nT per division) obtained from auroral ground-based stations during the interval 14:00-22:00 UT on day 73, 1993. The marked arrow at $\sim 16: 48$ UT corresponds to a distinct substorm onset. The expansion phase of the same substorm is detected on board GEOTAIL at $\sim 16: 52$ UT. The flow reversal at $\sim 18: 10$ UT may correspond to the recovery phase of this marked substorm. From top to bottom the stations are the Contwoyto Lake, Leirvogur observatory, MCQ, Cape Chelyskin and Tixie

positive excursion at the Leirvogur observatory (LRV) is produced by the eastward electrojet, because for this station the MLT $=0$ occurs at 00:15 UT. The Earthward flux detected at GEOTAIL during the interval 18:10-18:25 UT could well be associated with the recovery phase of this concrete substorm that started at $\sim 16: 48$ UT. The earliest detected energetic electrons were measured at $\sim 16: 52 \mathrm{UT}$, that is, only $\sim 4$ min after the substorm onset. The near-Earth neutral-line model for substorms suggests that in this case a new acceleration site must be formed in the near-Earth region (e.g., at $-20 \mathrm{R}_{\mathrm{E}}$ ) at $\sim 16: 48 \mathrm{UT}$, and predicts that a plasmoid must be formed in the near-Earth tail and subsequently propagated downtail. Indeed, a possible interpretation of the phenomenon of tailward-to-Earthward flow reversal may be the tailward retreat of a plasmoid downstream of spacecraft. The time-delay between the substorm onset and the appearance of Earthward flux is $\sim 82$ min. If the downtail propagation velocity of the acceleration site is constant, then the speed is $\sim 205 \mathrm{~km} \mathrm{~s}^{-1}$ (i.e., $160 \mathrm{R}_{\mathrm{E}}$ divided by $82 \mathrm{~min}$ ). Angelopoulos et al. (1996) found a speed $75-115 \mathrm{~km} \mathrm{~s}^{-1}$ out of 14 substorms, while GEOTAIL was located at $-90 \mathrm{R}_{\mathrm{E}}$.
If we compare the two results then the assumption of constant speed of plasmoid propagation may be incorrect. Instead, the plasmoid seems to have accelerated moving downtail. In the case-study of day 73 of 1993, a tailward-propagating plasmoid could not be detected on the basis of a bipolar signature of north-then-south deviation along the $B_{z}$ component of the magnetic field because the spacecraft crosses the neutral sheet. Strong evidence of a plasmoid formation may be the isotropic angular distributions of energetic electrons at the CPS region detected during the first transition from the north to the south lobe, as well as during the brief excursion to the CPS. The objection may arise that if the plasmoid formation has indeed taken place, then its passage over the spacecraft remaining at lobe magnetic field lines should be manifested as the bipolar signature associated with a traveling compression region (TCR) (Slavin et al., 1993). But we fail to determine such a distinct observational feature during the interval that spans the time between the reversal of energetic ion fluxes.

The tailward propagation of a plasmoid has been considered the cause of the spacecraft motion from one lobe into the plasma sheet and then back into the same lobe again, as if a bulge in the plasma sheet had passed by (Baker et al., 1987). But here we did not have this typical case. Instead, it was the flapping of the plasma sheet that resulted in entries of the spacecraft into the plasma sheet.

The energetic proton bursts at the PSBL are highly collimated along the magnetic field and this is illustrated via the top angular distribution of Fig. 13 corresponding to the entry interval 16:56:02-16:57:45 UT into the plasma sheet of day 73, 1993. The ratio of tailward-toEarthward flux is higher than three orders of magnitude and the vector magnetic field deviates $7^{\circ}$ from Sunward. It is not observable any dawn-dusk anisotropy due to $\boldsymbol{B} \times \nabla \boldsymbol{j}$ flow, where $\nabla \boldsymbol{j}$ stands for the energetic-proton density gradient. Sarafopoulos and Sarris (1991a) used extensively the angular distributions as a powerful tool for probing the near-Earth PSBL stucture, but in the present case no one angular distribution upon the entry into the plasma sheet shows a detectable duskward flux (even if the distributions are scrutinized with time resolution as high as $3 \mathrm{~s}$ ). Consequently, it is impossible to be estimated any spacecraft velocity relative to the PSBL. During the exit at $\sim 17: 22$ UT a gradient anisotropy of the just-receding layer is seen, but not so clearly as to derive a boundary velocity. The second average angular distribution of $58-77-\mathrm{keV}$ protons shown in Fig. 13 corresponds to the CPS region from $17: 05$ to $17: 15$ UT of day 73,1993 . Although the particles continue streaming tailward, a less anisotropic character (as compared with that at the PSBL) is apparent. In this case the vector magnetic field is highly variable throughout the interval and is thus eliminated.

The six successive PSBL crossings which occurred during the interval 16:45-18:20 UT of day 73, 1993, seem to be associated with a magnetospheric substorm having an onset just before the first crossing. The combined effect of neutral-sheet flapping and a tailwardmoving X-type magnetic field neutral line is given via 
Log. Scale . 58-77 keV Protons Overall Angular Distribution from 16:56:02 to $16: 57: 45$ UT (i.e., Fluxes at Entry)

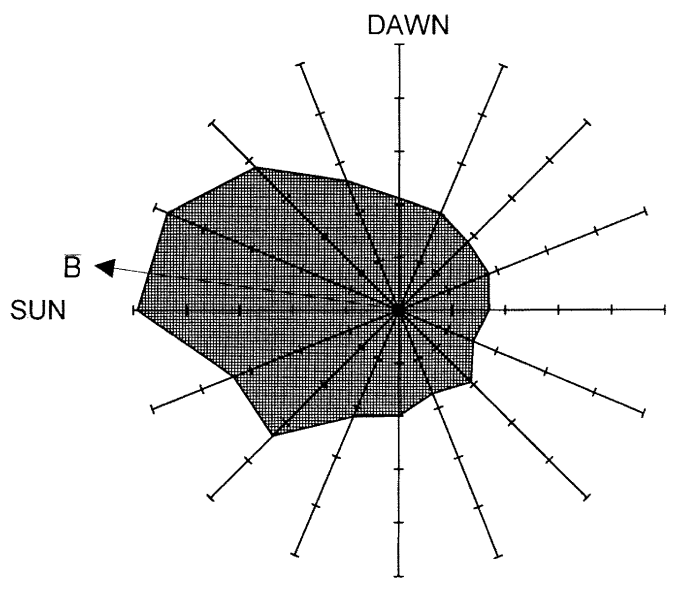

Log. Scale . 58-77 keV Protons.

Overall Angular Distribution From 17:05 to 17:15 (i.e., at the CPS)

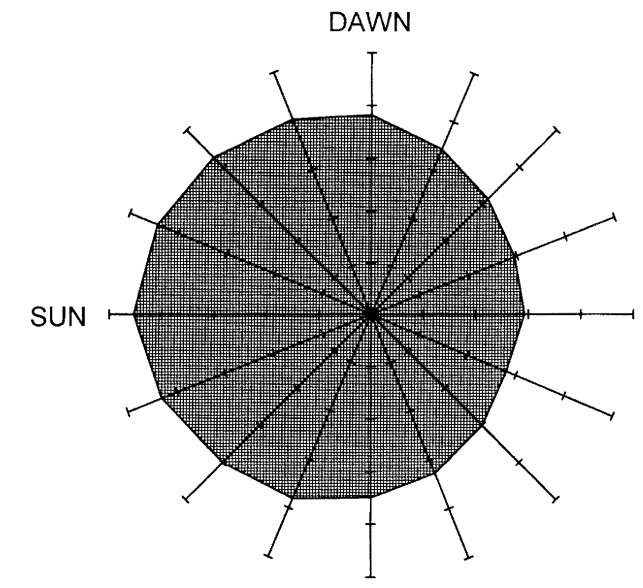

Fig. 13. Overall angular distributions at the PSBL and the CPS of the $58-77-\mathrm{keV}$ protons in logarithmic scale. The particles stream along the magnetic field lines although at the CPS a less anisotropic character is apparent. The dominant direction of flux is tailward and the dawn points-upward

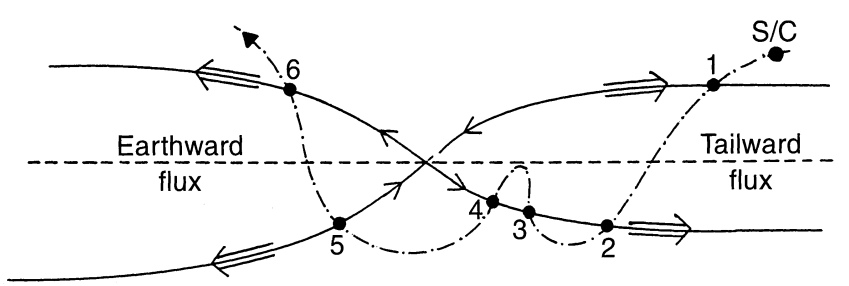

Fig. 14. The combined effect of neutral-sheet flapping during the interval 16:45-18:20 UT of day 73, 1993 and a tailward-moving Xtype magnetic field line is given in this sketch illustrating the GEOTAIL (S/C) trajectory. The six successive crossings of the PSBL occurred at $\sim 180 \mathrm{R}_{\mathrm{E}}$ and are characterized by streaming ions in a direction emphasized by large arrows. The first crossing occurred just after the substorm onset at $\sim 16: 48$ UT

the sketch of Fig. 14. The large arrows show the direction of streaming ions, while the solid ones determine the separatrices. The Earthward-streaming ions are associated with closed magnetic field lines, while the tailward-streaming ions occurred with an open geometry. In conclusion, the flux reversal may well be interpreted in terms of a neutral line moving beyond $\sim 180 \mathrm{R}_{\mathrm{E}}$.

The Earthward fluxes of energetic electrons and ions are associated with relatively low intensities of energetic electrons, which concurs with Richardson et al. (1993), who concluded that bursts of energetic electrons fall off with downtail distance such that they are nearly absent beyond $\sim 100 \mathrm{R}_{\mathrm{E}}$ from the Earth. Also, contrary to expectations, in this work the Earthward ion fluxes occurred with no positive $B_{z}$ component of the magnetic field.

The inverse velocity dispersion (IVD) effect (i.e., lowenergy, $\sim 300-\mathrm{keV}$ ions are detected 10-20 s before the high-energy, $\sim 1-\mathrm{MeV}$ ions) observed with impulsive bursts of energetic particles inside the Earth's plasma sheet and PSBL (see Sarris and Axford, 1979; Sarafopoulos and Sarris, 1988; Taktakishvili et al., 1993), and interpreted as the growth rate of the accelerating source, must be disconnected with the velocity dispersions displayed in this work because of their striking difference in time-scale. In addition, the IVDs occurred irrespectively of entries into and exits from the plasma sheet.

Sarris et al. (1996) on one case-study of the velocity dispersion effect concluded that the sequence of events during the dispersive onset of energetic particle burst presented a self-consistent picture, being the result of time of flights from a semipermanent source (of energetic particles). In this study the incorporation of data sets with energies as low as $\sim 9.4-\mathrm{keV}$ protons, in parallel with the fact that reverse velocity dispersions accompany exits from the plasma sheet, leads us to suggest the spatial structure of the plasma sheet as the cause producing the velocity dispersion effect.

In summary, the data presented here allow us to derive the following major findings:

1. The energetic electrons are freely streaming almost exclusively at the PSBL and show peak fluxes at lobe field lines or at the PSBL. Conversely, when higher fluxes of energetic electrons occur at CPS regions, then they show isotropic angular distributions, which are presumably associated with closed magnetic field lines.

2. The ions are spatially separated in energy-dependent zones of tailward-jetting-particles. The lower energies occupy the innermost depths of the plasma sheet. A very accurate velocity filter effect has taken place.

3. The proton layer is confined within the energetic electron layer, the alpha particle layer is confined within the proton layer and the oxygen layer is confined within the alpha particle layer, if the same energy level for all the species is considered.

4. During the transition of the spacecraft from the north to the south lobe, "normal velocity dispersion" is detected during the entry and "reverse velocity dispersion" during the exit from the plasma sheet. The entire plasma sheet appears to display a "mirror-image" in spatial structure, with the axis of symmetry being the neutral sheet. 
5. We have determined the time-delay between the substorm onset on Earth and the first detected energetic electron flux at $\sim 180 \mathrm{R}_{\mathrm{E}}$ to be $\sim 4 \mathrm{~min}$. The tailward-toEarthward flow reversal occurred $82 \mathrm{~min}$ after the substorm onset. If we assume a constant plasmoid velocity then it is $205 \mathrm{~km} \mathrm{~s}^{-1}$.

Acknowledgements. We are grateful to G. Rostoker for providing us with ground magnetometer data from the CANOPUS instrument array. Also, we thank Dr. T. Saemundsson of Leirvogur (LRV) observatory, as well as Dr. S. Solovyev and IFKIA, Russian Academy of Science for the use of the data from stations TIX and CHD.

Topical Editor K. H. Glaßmeier thanks B. Mauk and another referee for their help in evaluating this paper.

\section{References}

Andrews, M. K., E. Keppler, and P. W. Daly, Plasma sheet motions inferred from medium-energy ion measurements, J. Geophys. Res., 86, 7543-7556, 1981 .

Angelopoulos, V., A. T. Lui, R. W. McEntire, D. J. Williams, S. P. Christon, M. Nakamura, H. Kusaka, T. Mukai, S. Kokubun, T. Yamamoto, G. D. Reeves, E. Friis-Christensen, and W. J. Hughes, Anisotropy reversals in the distant magnetotail and their association with magnetospheric substorms, J. Geomagn. Geoelectr., 48, 629-648, 1996.

Baker, D. N., R. C. Anderson, R. D. Zwickl, and J. A. Slavin, Average plasma and magnetic field variations in the distant magnetotail associated with near-Earth substorm effects, $J$. Geophys. Res., 92, 71-81, 1987.

Daly, P. W., T. R. Sanderson, and K. -P. Wenzel, Survey of energetic $(E>35 \mathrm{keV})$ ion anisotropies in the deep geomagnetic tail, J. Geophys. Res., 89, 10733-10739, 1984.

De Coster, R. J., and L. A. Frank, Observations pertaining to the dynamics of the plasma sheet, J. Geophys. Res., 84, 5099-5121, 1979.

Eastman, T. E., L. A. Frank, W. K. Peterson, and W. Lennartsson, The plasma sheet boundary layer, J. Geophys. Res., 89, 15531572, 1984.

Eastman, T. E., L. A. Frank, and C. Y. Huang, The boundary layers as the primary transport regions of the Earth's magnetotail, $J$. Geophys. Res., 90, 9541-9560, 1985.

Kokubun, S., T. Yamamoto, M. H. Acuna, K. Hayashi, K. Shiokawa, and H. Kawano, The GEOTAIL magnetic field experiment, J. Geomagn. Geoelectr., 46, 7-21, 1994.

Lui, A. T. Y., L. A. Frank, K. L. Ackerson, C. I. Meng, and S. -I. Akasofu, Plasma flows and magnetic field vectors in the plasma sheet during substorms, J.Geophys. Res., 83, 3849-3858, 1978.

Mobius, E., F. M. Ipavich, M. Scholer, G. Gloeckler, D. Hovestadt, and B. Klecker, Observations of a nonthermal ion layer at the plasma sheet boundary during substorm recovery, J.Geophys. Res., 85, 5143-5148, 1980.

Parks, G. K., M. McCarthy, R. J. Fitzenreiter, J. Etcheto, K. A. Anderson, R. R. Anderson, T. E. Eastman, L. A. Frank, D. A. Gurnett, C. Huang, R. P. Lin, A. T. Y. Lui, K. W. Ogilvie, A. Pedersen, H. Reme, and D. J. Williams, Particle and field characteristics of the high-latitude plasma sheet boundary layer, J. Geophys. Res., 89, 8885-8906, 1984.

Parks, G. K., R. Fitzenreiter, K. W. Ogilvie, C. Huang, K. A. Anterson, J. Dandouras, L. Frank, R. P. Lin, M. McCarthy, H. Reme, J. A. Sauvaud, and S. Werden, Low-energy particle layer outside of the plasma sheet boundary, J. Geophys. Res., 97, 2943-2954, 1992.
Richardson, I. G., and S. W. H. Cowley, Plasmoid-associated energetic ion bursts in the deep geomagnetic tail: properties of the boundary layer, J. Geophys. Res., 90, 12133-12158, 1985.

Richardson, I. G., C. J. Owen, J. A. Slavin, and T. T. von Rosenvinge, Energetic ( $>0.2 \mathrm{MeV}$ ) electron bursts observed by ISEE 3 in the deep $\left(<240 \mathrm{R}_{\mathrm{E}}\right)$ geomagnetic tail, J. Geophys. Res., 98, 13441-13451, 1993.

Sarafopoulos, D. V., and E. T. Sarris, Inverse velocity dispersion of energetic particle bursts inside the plasma sheet, Planet. Space Sci., 36, 1181-1199, 1988.

Sarafopoulos, D. V., and E. T. Sarris, Substorm associated energetic ion $(\mathrm{E} \cong 45 \mathrm{keV})$ flows at the plasma sheet boundary layer: a dawn-dusk flow reversal, Planet. Space Sci., 38, 12511266, 1990.

Sarafopoulos, D. V., and E. T. Sarris, Determination of the primary region of access of energetic particles from their source in the Earth's plasma sheet, Ann. Geophysicae, 9, 429-441, 1991a.

Sarafopoulos, D. V., and E. T. Sarris, Long-period standing waves at the plasma sheet boundary layer region observed by ISEE-1, Ann. Geophysicae, 9, 333-347, 1991 b.

Sarris, E. T., and W. I. Axford, Energetic protons near the plasma sheet boundary, Nature, 227, 460, 1979.

Sarris, E. T., Angelopoulos, R. W. McEntire, D. J. Williams, S. M. Krimigis, A. T. Y. Lui, E. C. Roelof, and S. Kokubun, Detailed observations of a burst of energetic particles in the deep magnetotail by GEOTAIL, J. Geomagn. Geoelectr., 48, 649656, 1996.

Schindler, K., D. N. Baker, J. Birn, E. W. Hones Jr, J. A. Slavin, and A. B. Galvin, Analysis of an extended period of earthward plasma sheet flow at $\sim 220 \mathrm{R}_{\mathrm{E}}$ : CDAW 8, J. Geophys. Res., 94, 15177-15188, 1989.

Scholer, M., A review of the ISEE-3 geotail suprathermal ion and electron results, Planet. Space Sci., 34, 915-930, 1986.

Scholer, M., D. N. Baker, G. Gloeckler, B. Klecker, F. M. Ipavich, T. Terasawa, B. T. Tsurutani, and A. B. Galvin, Energetic particle beams in the plasma sheet boundary layer following substorm expansion: simultaneous near-Earth and distant tail observations, J. Geophys. Res., 91, 4277-4286, 1986.

Scholer, M., B. Klecker, D. Hovestadt, G. Gloeckler, F. M. Ipavich, A. B. Galvin, D. N. Baker, and B. T. Tsurutani, Energetic ion and electron beams at the plasma-sheet boundary in the distant tail, in Magnetotail physics, Ed. T.Y. Lui, Johns Hopkins University Press, Baltimore, 245-249, 1987.

Slavin, J. A., M. F. Smith, E. L. Mazur, D. N. Baker, E. W. Hones Jr, T. Iyemori, and E. W. Greenstadt, ISEE-3 Observations of traveling compression regions in the Earth's magnetotail, $J$. Geophys. Res., 98, 15425-15446, 1993.

Spjeldvik, W. N., and T. A. Fritz, Energetic ion and electron observations of the geomagnetic plasma sheet boundary layer: three-dimensional results from ISEE-1, J. Geophys. Res., 86, 2480-2486, 1981.

Taktakishvili, A. L., L. M. Zelenyi, E. T. Sarris, R. E. Lopez, and D.V. Sarafopoulos, Temporal dispersion structures of proton and electron bursts in the Earth's magnetotail, Planet. Space Sci., 41, 461-467, 1993.

Williams, D. J., Energetic ion beams at the edge of the plasma sheet: ISEE-1 observations plus a simple explanatory model, $J$ Geophys. Res., 86, 5507-5518, 1981.

Williams, D. J., R. W. McEntire, C. Schemm II, A. T. Y. Lui, G. Gloeckler, S. P. Christon, and F. Gliem, GEOTAIL energetic particles and ion composition instrument, J. Geomagn. Geoelectr., 46, 39-57, 1994.

Zwickl, R. D., D. N. Baker, S. J. Bame, W. C. Feldman, J. T. Gosling, E. W. Hones Jr, D. J. McComas, B. T. Tsurutani, and J. A. Slavin, Evolution of the Earth's distant magnetotail: ISEE-3 electron plasma results, J. Geophys. Res., 89, 1100711012, 1984. 